\title{
TUBERCULOSIS
}

\section{Investigation of mycobacterial colonisation and invasion of the respiratory mucosa}

\author{
A M Middleton, M V Chadwick, A G Nicholson, A Dewar, C Feldman, R Wilson
}

Thorax 2003;58:246-251

Background: The pathogenesis of Mycobacterium avium complex and Mycobacterium tuberculosis in the respiratory tract is poorly understood, as are the reasons for their differing virulence. We have previously shown that their initial adherence to the mucosa is identical.

Methods: The interaction of $M$ avium complex, $M$ tuberculosis, and $M$ smegmatis with human respiratory tissue was investigated in an organ culture model with an air interface. Tissue was infected for intervals up to 14 days and assessed by scanning electron microscopy for adherent bacteria or cultured for recoverable bacteria.

See end of article for authors' affiliations

Results: The mean number of adherent bacteria/ $\mathrm{mm}^{2}$ (and the viable count of macerated tissue, cfu/ $\mathrm{ml}$ ) at 15 minutes, 3 and 24 hours, 7 and 14 days were: $M$ avium complex 168 (153), 209 (136), 289 (344), 193 (313), 14140 (16544); M tuberculosis 30 (37), 39 (23), 48 (53), 1 (760), 76

Correspondence to: Dr R Wilson, Host Defence Unit, Royal Brompton Hospital, Sydney Street London SW3 6NP, UK: r.wilson@rbh.nthames.nhs.uk

Revised version received 25 September 2002 Accepted for publication 18 November 2002 (2186); $M$ smegmatis 108 (176), 49 (133), 97 (81), 114 (427), 34 (58), (n=6). There was no significant change in morphology between infected and uninfected tissue or tissue infected with the different species over 14 days. The number of $M$ avium complex on the mucosa and recovered from tissue increased over time $(p=0.03)$. M tuberculosis decreased on the surface, but recoverable bacteria increased $(p=0.01)$. M smegmatis numbers on the mucosa and recovered from tissue decreased. Sectioned tissue showed $M$ avium complex and $M$ tuberculosis in submucosal mucus glands and $M$ tuberculosis penetrating epithelial cells in one experiment.

Conclusions: The initial adherence to the mucosa of the three species was similar, but after 14 days they varied in their interaction with the tissue in a manner compatible with their pathogenicity.
M ycobacterium avium complex and Mycobacterium tuberculosis both cause pulmonary disease in humans. $M$ avium complex is generally referred to as an opportunistic pathogen since it usually infects patients with established lung damage, including scarring from previous tuberculosis, cystic fibrosis and other forms of bronchiectasis, pneumoconiosis, and chronic obstructive pulmonary disease. ${ }^{1-4} M$ avium complex can also cause primary infections in patients without any history of respiratory illness, ${ }^{5-9}$ and these have been reported with increasing frequency in recent years. ${ }^{561011} \mathrm{In}$ developed countries endobronchial or mediastinal infections $^{12}{ }^{13}$ and solitary pulmonary nodules ${ }^{14}$ are increasingly common. Chronic infection of the airway causes bronchiectasis. ${ }^{15}$ In addition, parenchymal infections manifesting as pneumonitis or cavitary disease have been reported. ${ }^{\circ}$

$M$ tuberculosis is an obligate human pathogen transmitted from patient to patient in respiratory secretions and aerosols. Interaction with the alveolar macrophage is the most important mechanism in the disease process but there may be others because, in one study, resident macrophages did not bind $M$ tuberculosis efficiently. ${ }^{16}$ Endobronchial tuberculosis may develop via mucosal surface infection in smear positive cases by lymphatic extension from a peribronchial focus or by miliary spread. ${ }^{17}$ Thompson and Kent concluded that most cases of endobronchial disease were the result of the surface implantation of $M$ tuberculosis and that lack of apparent damage seen on bronchoscopy is possibly due to rapid healing and repair of the mucosal surface. ${ }^{17}$ An alternative explanation might be uptake of $M$ tuberculosis by specialised epithelial cells which might not necessarily result in damage to the cell. ${ }^{18-20}$

The pathogenetic mechanisms of $M$ avium complex and $M$ tuberculosis in the respiratory tract are poorly understood, as are the reasons for their differing virulence. We have previously reported the initial interaction of $M$ avium complex,
$M$ tuberculosis, and $M$ smegmatis with the respiratory mucosa of human respiratory tissue organ cultures having an air interface. ${ }^{21} 22$ These experiments showed similar adherence of the three species, predominantly to mucus with a fibrous appearance and to fibronectin in extracellular matrix (ECM) exposed when the epithelium was damaged. The results of these studies showed that the virulence of the mycobacterial species did not influence their initial adherence to the respiratory mucosa. We have therefore investigated the adherence to and recovery from organ cultures of $M$ avium complex, $M$ tuberculosis, and $M$ smegmatis at intervals over 14 days incubation.

\section{METHODS}

\section{Bacteria}

A clinical strain of $M$ avium complex previously described, ${ }^{21} M$ tuberculosis $\mathrm{H}_{3} 7_{\mathrm{RV}}$ (ATCC 27294), and $M$ smegmatis strain $\mathrm{Mc}^{2} 155$ were used in the study. ${ }^{23}$ The mean viable counts of the inocula $(2 \mu \mathrm{l})$ were calculated as previously described. ${ }^{21}$ The sizes of the inocula for $M$ avium complex, $M$ tuberculosis, and $M$ smegmatis were $9.3 \times 10^{7} \mathrm{cfu} / 2 \mu \mathrm{l}$ (range $\left.6.6 \times 10^{7}-1.5 \times 10^{8}\right), 7.8$ $\times 10^{7} \mathrm{cfu} / 2 \mu \mathrm{l}\left(\right.$ range $\left.7.1 \times 10^{7}-9.1 \times 10^{7}\right)$, and $3.8 \times 10^{8} \mathrm{cfu} / 2 \mu \mathrm{l}$ (range $3.1 \times 10^{8}-5.0 \times 10^{8}$ ), respectively. There was no significant difference between the number of bacteria in the inocula of the different series of experiments.

\section{Organ culture}

The organ culture with an air interface used in these experiments has been described previously. ${ }^{21-26}$ Human nasal turbinate was obtained from patients undergoing surgery for nasal obstruction and human bronchial tissue from patients undergoing tumour resection. Tissue was used in experiments with the written permission of the patients and the protocol of tissue removal and its use in this study was approved by the Royal Brompton Hospital ethics committee. 


\section{Experimental design}

Each experiment required 80 pieces of dissected tissue. Organ cultures were studied at five time intervals with the three species matched with an uninfected control; separate organ cultures were processed for scanning electron microscopy (SEM), culture, light microscopy, and transmission electron microscopy (TEM). Two $\mu \mathrm{l}$ of a washed bacterial suspension ( $M$ avium complex, $M$ tuberculosis or $M$ smegmatis) in phosphate buffered saline (PBS) were gently pipetted onto the surface of the organ culture. An uninfected control organ culture, inoculated with PBS alone, was also harvested at each time interval. Uninfected and infected tissues were placed at $37^{\circ} \mathrm{C}$ in $5 \% \mathrm{CO}_{2}$ for 15 minutes, 3 hours, 24 hours, 7 days, and 14 days. After harvesting from the organ cultures the tissue was washed by pipetting $100 \mu \mathrm{l}(\times 3)$ of sterile PBS onto the mucosal surface in order to remove unattached bacteria.

\section{Preparation and assessment of tissue for SEM}

The method used for tissue preparation and assessment by SEM has been described previously..$^{24-26}$ The tissue was assessed for the percentage of the surface occupied by four main mucosal features: mucus, ciliated cells, unciliated cells, and damaged mucosa. The mucosal morphology was compared over the five time intervals and between infected and uninfected tissue. The number of bacteria seen on the tissue was counted for the entire mucosa. The actual area of the mucosa was calculated taking into account the $\times 25$ magnification of the microscope and the $\times 2.5$ visual display unit magnification. Using this figure and the number of bacteria adherent to the mucosa, the number of bacterial cells per $\mathrm{mm}^{2}$ was calculated for each species at each incubation time.

\section{Calculation of viable counts from macerated tissue}

Tissue was removed from the organ culture taking care to remove all surrounding agar. One hundred $\mu \mathrm{l}$ of sterile PBS was placed in a sterile graduated centrifuge tube and the meniscus was marked on the side of the tube. This procedure was carried out for all centrifuge tubes and for each separate experiment to compensate for variation in tubes and ambient temperature. Tissue was placed in the PBS and the displacement volume was estimated by the amount of PBS required to be removed to return the meniscus to the marked level. The tissue was then placed in $1 \mathrm{ml}$ sterile PBS in a homgeniser grinder tube and homogenised three times for 10 seconds, with a 10 second interval between each spin to prevent temperature build up in the tissue/PBS due to friction. Ten-fold dilutions of the homogenate were then performed down to $10^{-6}$. Viable counts were performed as previously described $^{21}$ and the number of bacteria recovered per $\mu \mathrm{l}$ of tissue was calculated using the displacement volume of the tissue.

\section{Preparation and assessment of tissue for light microscopy}

Tissue was fixed in 4\% formaldehyde, dehydrated, and embedded in paraffin. Sections were made to study the location of the mycobacteria. Five $\mu \mathrm{m}$ sections were cut and stained with Ziehl-Neelsen stain. Sections from the same tissue were also cut, fixed, and stained with haematoxylin and eosin.

\section{Preparation and assessment of tissue for TEM}

Tissue was initially fixed in $2.5 \%$ glutaraldehyde and then post fixed in $1 \%$ osmium tetroxide, dehydrated, and embedded in araldite. Ultrathin $(80 \mathrm{~nm})$ sections were then cut and used to look for mycobacteria on the surface and within the mucosa by TEM.

\section{Statistical analysis}

Comparison of the percentages of the surface area occupied by mucosal features for infected and control tissue, the number of adherent bacteria, and the number of bacteria recovered from macerated tissue at different time intervals were analysed using Wilcoxon's matched signed rank test. p values of $\leqslant 0.05$ were judged to be significant.

\section{RESULTS}

Comparison of the number of mycobacteria observed on the mucosal surface by SEM with the number recovered from macerated tissue

Table 1 shows the morphometric analysis of the four main mucosal features: mucus, damaged epithelium, ciliated, and unciliated cells. Incubation of the organ cultures for up to 14 days had no significant effect on the morphological features of the tissue. There was no significant difference between organ cultures inoculated with the three species of mycobacteria, nor between infected tissue and uninfected controls (data not shown).

Figure 1 shows the mean number of bacteria adhering to the mucosal surface of organ cultures (bacteria $/ \mathrm{mm}^{2}$ ) and the

Table 1 Morphometric analysis of the mucosa of Mycobacterium avium complex (MAC), Mycobacterium tuberculosis (TB), and Mycobacterium smegmatis (SM) infected organ cultures $(n=6)$

\begin{tabular}{|c|c|c|c|c|c|c|c|c|c|c|c|c|c|c|c|c|c|c|c|c|}
\hline & \multicolumn{5}{|c|}{ Mucus } & \multicolumn{5}{|c|}{ Damaged mucus } & \multicolumn{5}{|c|}{ Ciliated mucosa } & \multicolumn{5}{|c|}{ Unciliated mucosa } \\
\hline & $15 \mathrm{~m}$ & $3 \mathrm{~h}$ & $24 \mathrm{~h}$ & $7 d$ & $14 \mathrm{~d}$ & $15 \mathrm{~m}$ & $3 \mathrm{~h}$ & $24 \mathrm{~h}$ & $7 d$ & $14 \mathrm{~d}$ & $15 \mathrm{~m}$ & $3 \mathrm{~h}$ & $24 \mathrm{~h}$ & $7 d$ & $14 \mathrm{~d}$ & $15 \mathrm{~m}$ & $3 \mathrm{~h}$ & $24 \mathrm{~h}$ & $7 d$ & $14 \mathrm{~d}$ \\
\hline \multicolumn{21}{|l|}{ MAC } \\
\hline Mean & 22.7 & 19.7 & 15.1 & 18.3 & 10.8 & 24.0 & 18.6 & 20.5 & 15.3 & 37.4 & 14.2 & 16.9 & 12.4 & 19.6 & 12.2 & 39.1 & 44.8 & 52.0 & 46.9 & 39.6 \\
\hline SE & 9.2 & 6.4 & 7.1 & 2.4 & 5.5 & 12.6 & 10.3 & 11.0 & 6.2 & 16.9 & 7.8 & 7.5 & 7.5 & 9.3 & 4.3 & 11.9 & 10.2 & 12.8 & 6.8 & 10.3 \\
\hline Median & 15.5 & 16.2 & 13.2 & 19.1 & 6.1 & 14.8 & 12.5 & 12.7 & 14.8 & 17.6 & 8.9 & 10.4 & 9.6 & 13.4 & 14.0 & 40.8 & 45.7 & 42.2 & 42.4 & 43.7 \\
\hline $\mathrm{n}$ & $5 / 6$ & $5 / 6$ & $5 / 6$ & $6 / 6$ & $4 / 6$ & $5 / 6$ & $5 / 6$ & $5 / 6$ & $4 / 6$ & $5 / 6$ & $6 / 6$ & $6 / 6$ & $6 / 6$ & $5 / 6$ & $6 / 6$ & $6 / 6$ & $6 / 6$ & $6 / 6$ & $6 / 6$ & $6 / 6$ \\
\hline \multicolumn{21}{|l|}{ TB } \\
\hline Mean & 12.8 & 18.5 & 17.4 & 13.5 & 16.6 & 19.3 & 28.7 & 21.2 & 25.3 & 29.0 & 20.3 & 21.6 & 15.8 & 11.8 & 16.3 & 47.6 & 31.2 & 45.6 & 49.4 & 38.1 \\
\hline SE & 5.1 & 6.5 & 3.7 & 4.7 & 5.7 & 8.8 & 13.1 & 11.4 & 11.2 & 19.4 & 18.6 & 16.2 & 14.3 & 8.5 & 8.0 & 7.0 & 7.8 & 11.3 & 6.8 & 9.0 \\
\hline Median & 11.6 & 16.4 & 13.6 & 14.5 & 11.3 & 14.7 & 16.9 & 14.1 & 17.8 & 19.3 & 11.3 & 12.8 & 6.4 & 0 & 11.1 & 46.7 & 28.5 & 33.9 & 44.1 & 35.1 \\
\hline$n$ & $5 / 6$ & $4 / 6$ & $4 / 6$ & $4 / 6$ & $5 / 6$ & $5 / 6$ & $4 / 6$ & $4 / 6$ & $4 / 6$ & $5 / 6$ & $6 / 6$ & $5 / 6$ & $5 / 6$ & $6 / 6$ & $6 / 6$ & $6 / 6$ & $6 / 6$ & $6 / 6$ & $6 / 6$ & $6 / 6$ \\
\hline \multicolumn{21}{|l|}{ SM } \\
\hline Mean & 16.8 & 26.4 & 19.0 & 15.1 & 11.0 & 24.7 & 25.9 & 22.8 & 26.5 & 31.3 & 15.3 & 19.3 & 17.9 & 16.8 & 16.3 & 43.2 & 28.4 & 40.3 & 41.6 & 41.4 \\
\hline SE & 2.4 & 18.4 & 8.1 & 3.2 & 2.7 & 10.5 & 9.3 & 16.2 & 11.0 & 25.8 & 7.4 & 8.6 & 8.6 & 8.3 & 6.8 & 6.2 & 4.7 & 5.7 & 7.5 & 6.5 \\
\hline Median & 17.1 & 14.6 & 15.5 & 16.5 & 11.3 & 19.4 & 22.6 & 12.9 & 15.6 & 19.4 & 12.5 & 16.7 & 9.3 & 11.8 & 17.1 & 44.8 & 30.7 & 42.4 & 42.1 & 38.0 \\
\hline $\mathrm{n}$ & $5 / 6$ & $5 / 6$ & $5 / 6$ & $5 / 6$ & $5 / 6$ & $5 / 6$ & $5 / 6$ & $5 / 6$ & $5 / 6$ & $6 / 6$ & $6 / 6$ & $6 / 6$ & $5 / 6$ & $6 / 6$ & $5 / 6$ & $6 / 6$ & $6 / 6$ & $6 / 6$ & $6 / 6$ & $6 / 6$ \\
\hline
\end{tabular}

Figures are the percentage of the mucosal surface surveyed occupied by a particular feature. $n=n u m b e r$ of experiments in which the mucosal feature was observed out of six.

$15 \mathrm{~m}=15$ minutes; $3 \mathrm{~h}$ and $24 \mathrm{~h}=3$ and 24 hours; $7 \mathrm{~d}$ and $14 \mathrm{~d}=7$ and 14 days incubation time. 


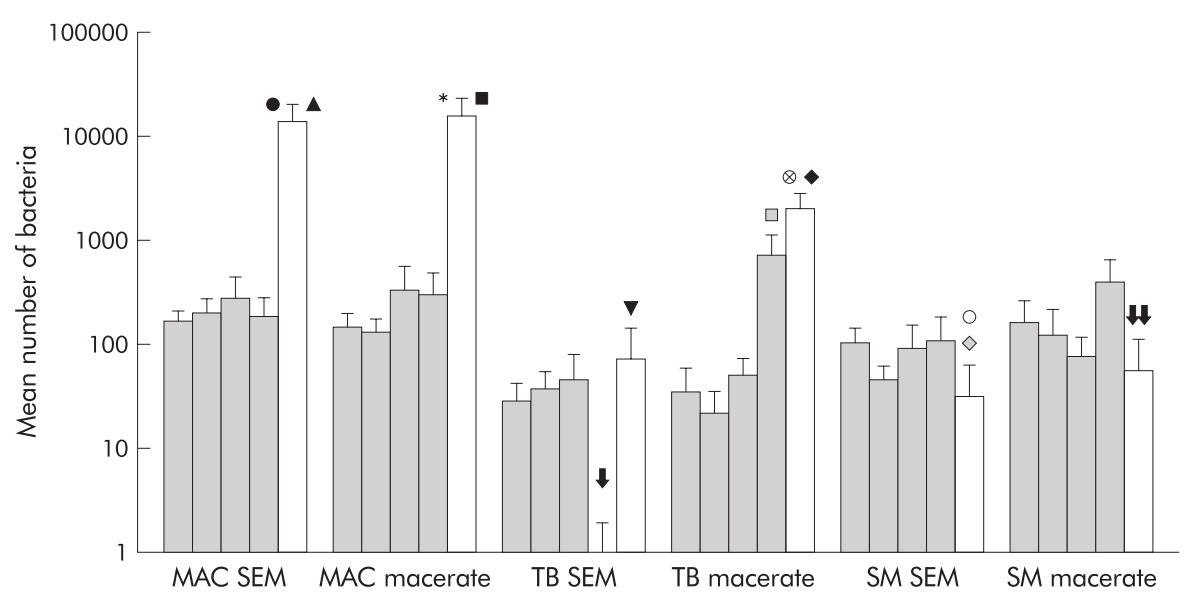

Figure 1 Mean number of Mycobacterium avium complex (MAC), $M$ tuberculosis (TB), and $M$ smegmatis (SM) adhering to the mucosal surface (scanning electron microscopy (SEM); bacteria $/ \mathrm{mm}^{2}$ ) or recovered from macerated tissue (macerate; cfu/ $\mu$ ) following incubation for 15 minutes, 3 hours, 24 hours, 7 days and 14 days (from left to right). $\bullet, 15$ minutes, 3 and 24 hours $v 14$ days ( $p=0.04$ ); $\boldsymbol{\Lambda}, 7$ days $v 14$ days $(p=0.03) ; *, 5$ minutes, 3 and 24 hours $v 14$ days ( $=0.03) ; 0$ days $v 14$ days ( $p<0.02) ; \downarrow, 15$ minutes, 3 and 24 hours $v 7$ days $(p=0.02) ; \nabla, 15$ minutes, 3 and 24 hours and 7 days $v 14$ days $(p=0.02) ; \square, 15$ minutes, 3 and 24 hours $v 7$ days $(p=0.03) ; \otimes, 15$ minutes, 3 and 24 hours $\vee 14$ days $(p=0.01) ;, 7$ days $\vee 14$ days $(p=0.02) ; 0,15$ minutes, 3 and 24 hours $v 14$ days $(p=0.02) ; \diamond, 7$ days $\vee 14$ days $(p=0.04) ; \downarrow \downarrow, 15$ minutes, 3 and 24 hours and 7 days $\vee 14$ days $(p=0.03)$. Results are expressed as the mean and standard error; $n=6$ (1 turbinate, 5 bronchial).

mean number of bacteria recovered from macerated tissue $(\mathrm{cfu} / \mu \mathrm{l})$ incubated for 15 minutes to 14 days. At 15 minutes all three mycobacterial species adhered in similar numbers to the same mucosal features, as previously described. ${ }^{21}{ }^{22}$ Bacteria adhered predominantly to ECM, mucus with a fibrous appearance, and were occasionally seen adhering to areas where extruded epithelial cells had degenerated exposing their contents, open tight junctions between cells, and to healthy unciliated epithelium. Bacteria did not adhere to mucus with a globular appearance, intact extruded cells, collagen fibres, or ciliated cells.

Table 2 shows the number of experiments in which $M$ avium complex, $M$ tuberculosis, and $M$ smegmatis were observed on the mucosal surface by SEM and were recovered from macerated tissue. $M$ tuberculosis was seen by SEM in two out of six experiments at 7 days incubation. However, the mean number of $M$ tuberculosis (1.2) does not register on fig 1 because of the scale of the $y$ axis. One experiment out of six analysed by SEM showed a large amount of mucosal damage ( $M$ tuberculosis, $58.3 \%$ of the mucosal surface at 14 days incubation; $M$ smegmatis, $62.4 \%$ at 14 days incubation) which exposed a large amount of ECM ( $M$ tuberculosis, 19.4\% of the mucosal surface at 14 days incubation; $M$ smegmatis, $17.3 \%$ ), and this was the one experiment in which $M$ tuberculosis and $M$ smegmatis were seen on the mucosal surface at this time point and the one experiment in which $M$ smegmatis was recovered from macerated tissue.

$M$ avium complex increased in numbers on the mucosal surface with time of incubation. There was a major increase in the numbers of $M$ avium complex seen by SEM after 14 days incubation compared with other time points. There was no significant difference in numbers observed by SEM when comparing counts between 15 minutes and 7 days incubation. $M$ avium complex recovered from macerated tissue increased with time of incubation. There was an increase in the number of bacteria recovered after 14 days incubation compared with other time points, but there was no significant difference in numbers recovered when comparing counts between 15 minutes and 7 days incubation. $M$ tuberculosis numbers decreased on the mucosal surface after 7 and 14 days incubation compared with earlier time points, whereas $M$ tuberculosis recovered from macerated tissue increased with time of incubation. There was an increase in the recovery of bacteria after 7 and 14 days incubation compared with earlier time points.
Table 2 Number of experiments in which Mycobacterium avium complex (MAC), Mycobacterium tuberculosis and Mycobacterium smegmatis were observed by scanning electron microscopy (SEM) and recovered from macerated tissue

\begin{tabular}{llll}
\hline Time & MAC & M tuberculosis & M smegmatis \\
\hline SEM & & & \\
15 minutes & $6 / 6$ & $5 / 6$ & $6 / 6$ \\
3 hours & $5 / 6$ & $5 / 6$ & $6 / 6$ \\
24 hours & $5 / 6$ & $2 / 6$ & $6 / 6$ \\
7 days & $3 / 6$ & $2 / 6$ & $3 / 6$ \\
14 days & $5 / 6$ & $1 / 6$ & $1 / 6$ \\
& & & \\
Macerated tissue & & & \\
15 minutes & $6 / 6$ & $6 / 6$ & $6 / 6$ \\
3 hours & $6 / 6$ & $6 / 6$ & $6 / 6$ \\
24 hours & $5 / 6$ & $6 / 6$ & $6 / 6$ \\
7 days & $6 / 6$ & $6 / 6$ & $4 / 6$ \\
14 days & $6 / 6$ & $6 / 6$ & $1 / 6$ \\
\hline
\end{tabular}

Data provided by a series of six separate experiments using tissue from a different donor for each experiment.

There was a decrease in the number of $M$ smegmatis seen on the mucosal surface and recovered from macerated tissue after 14 days compared with other time points.

The numbers of bacteria observed by SEM and recovered from macerated tissue at different times of incubation were compared for $M$ avium complex, $M$ tuberculosis and $M$ smegmatis. The significant differences were: $M$ avium complex was seen on the mucosal surface in greater numbers than $M$ tuberculosis and $M$ smegmatis after 14 days incubation; $M$ avium complex was recovered from macerated tissue in greater numbers than $M$ tuberculosis and $M$ smegmatis after 14 days incubation; and $M$ tuberculosis was recovered in greater numbers than $M$ smegmatis from macerated tissue after 14 days incubation.

The numbers of bacteria observed by SEM were compared with numbers recovered from macerated tissue for each mycobacterial species. There were more $M$ tuberculosis recovered from macerated tissue than adhering to the mucosal surface after 7 and 14 days, but not at earlier time points. There was no significant difference between numbers adhering to the mucosal surface and recovered from macerated tissue for $M$ avium complex and $M$ smegmatis for all incubation times. 


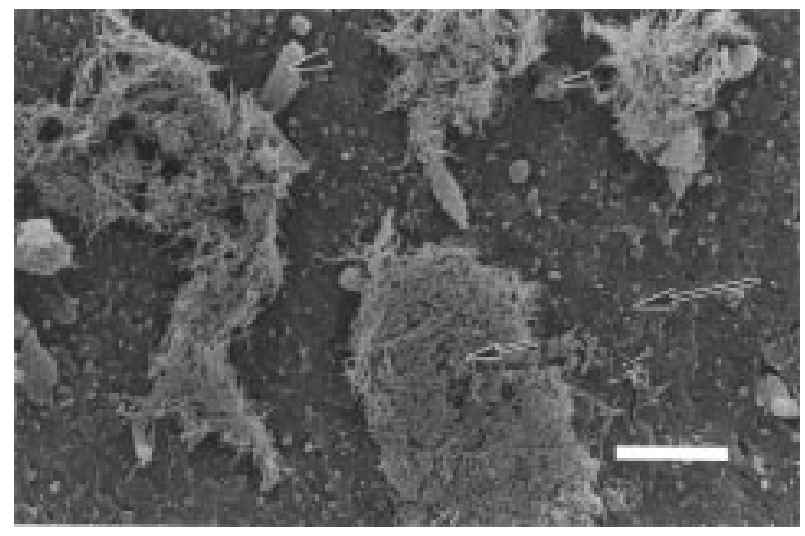

Figure 2 Mycobacterium avium complex adhering to organ culture after incubation for 14 days. The expected size of the mycobacteria used in this study is $1-4 \mu \mathrm{m} \times 0.5 \mu \mathrm{m}$. Magnification $\times 900$; scale bar $=20 \mu \mathrm{m}$. Large arrow=unciliated mucosa; small arrow= Mycobacterium avium complex; large arrowhead=extruded epithelial cell; small arrowhead=extracellular matrix.

Compared with 15 minutes incubation, SEM counts of $M$ avium complex were greater at 14 days indicating multiplication of the organism on the mucosal surface. Although $M$ avium complex was seen adhering to ECM and fibrous mucus, large numbers were seen disassociated from these features at 14 days. This suggests that $M$ avium complex initially adhered to fibrous mucus and ECM, but either replicated on the mucosal surface independently of them or obscured the features from observation by SEM because of the increased numbers of bacteria. This latter suggestion seems more likely as the bacteria were seen in sporadic "colonies" containing large numbers of organisms (fig 2).

\section{Light microscopy sections}

$M$ avium complex, $M$ tuberculosis, and $M$ smegmatis were seen adhering to the mucosa at 15 minutes, 3 and 24 hours, and 7 days. $M$ tuberculosis and $M$ smegmatis were not seen on the mucosal surface after 14 days incubation, whereas $M$ avium complex was seen in areas of damaged mucosa in increased numbers compared with other incubation times. $M$ avium complex was never observed adhering to intact mucosa in these experiments. $M$ avium complex and $M$ tuberculosis were seen within submucosal glands underlying damaged mucosa after 7 and 14 days incubation (fig 3). Figure 3 also shows invasion of the epithelium by $M$ tuberculosis within the gland.

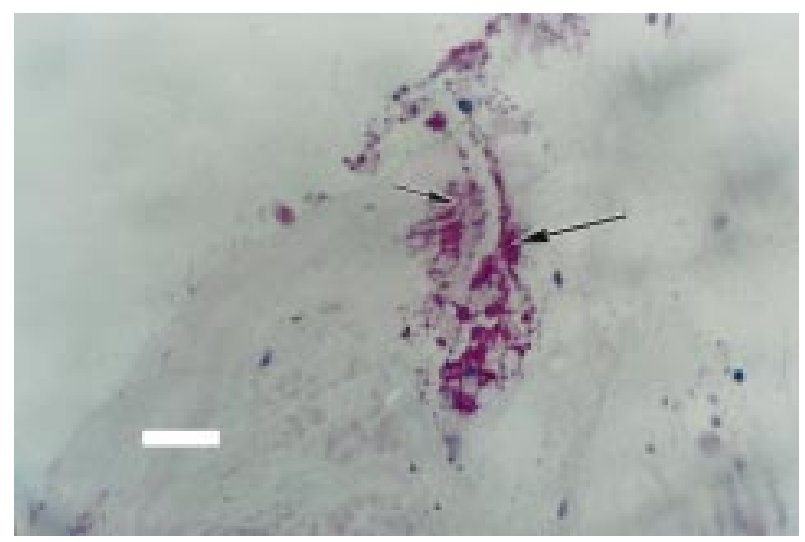

Figure 3 Ziehl-Neelsen stained paraffin embedded section showing Mycobacterium tuberculosis invasion of the epithelium within a submucosal gland after incubation for 14 days. Magnification $\times 258$; scale bar $=50 \mu \mathrm{m}$. Large arrow=Mycobacterium tuberculosis; small arrow=Mycobacterium tuberculosis within the tissue surrounding the submucosal gland.

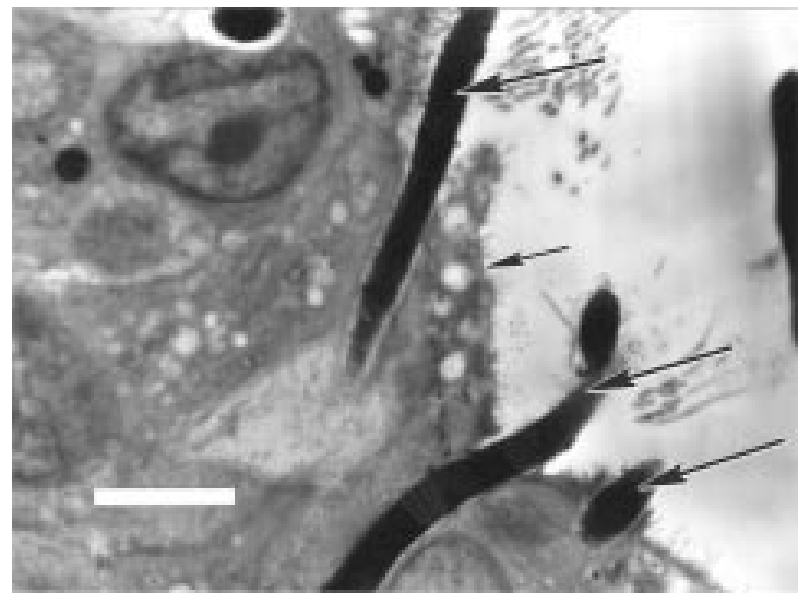

Figure 4 Transmission electron microscopy section showing Mycobacterium tuberculosis penetrating unciliated respiratory mucosa. Magnification $\times 20000$; scale bar $=1 \mu \mathrm{m}$. Large arrow=Mycobacterium tuberculosis; small arrow=mucosal surface.

They were, however, not seen in submucosal glands underlying intact mucosa. M smegmatis was never seen within submucosal mucus glands.

\section{TEM sections}

The solitary experiment in which mycobacteria were seen by TEM showed $M$ tuberculosis entering a healthy epithelial cell (fig 4). The observation of $M$ tuberculosis penetration of epithelial cells was not repeated in paraffin embedded light microscopy sections or by SEM analysis.

\section{DISCUSSION}

Interaction with the respiratory mucosa is likely to be a key event in the pathogenesis of $M$ avium complex which causes chronic airways infection and granulomatous inflammation leading to bronchiectasis, whereas in tuberculosis the primary pathology is in the alveoli and endobronchial disease is less common as the incidence of prolonged smear positive cases declines. $M$ smegmatis is an environmental species which is generally considered non-pathogenic. Infection of organ cultures with $M$ avium complex and $M$ tuberculosis did not affect mucosal morphology over 14 days incubation despite, in the case of $M$ avium complex, a large increase in the numbers of bacteria on the mucosal surface and, in the case of $M$ tuberculosis, invasion of the tissue. This observation might be because the mycobacteria do not secrete exotoxins, endotoxins, or tissue necrotising enzymes to aid their infectivity. However, it does raise the question as to how $M$ tuberculosis in particular invades the tissue. The adherence of $M$ avium complex and $M$ tuberculosis to open tight cell junctions may represent a potential route of infiltration into submucosal tissues. Since this site of adherence was observed at 15 minutes, it seems likely that mycobacteria took advantage of open tight junctions already present on the mucosa. Phagocytic cells are another potential route of invasion but were not observed on the surface of organ cultures. This could reflect the lack of host circulation from which these cells could migrate, or they may have been washed from the surface during preparation of tissue for microscopic analysis.

Mucosal penetration was not observed by SEM analysis of the mucosal surface of organ cultures, but mycobacteria did adhere to normal unciliated epithelial cells and, in a single experiment ( fig 4), endocytosis was observed by TEM. Clark et $a l^{27}$ used confocal microscopy to investigate the invasion of Yersinia pseudotuberculosis through murine Peyer's patch M cells with the aim of quantitating the relative contribution of $\mathrm{M}$ cells to intestinal invasion by $Y$ pseudotuberculosis. We propose 
that future studies of mycobacteria could employ a similar technique to investigate invasion of human airway tissue. Labelling of the mycobacteria with luciferase reporter constructs, as described by Snewin and colleagues, ${ }^{28}$ may also help to visualise bacteria penetrating the tissue.

The SEM surface counts and recoverable bacteria from macerated tissue showed that there was no difference in the numbers of mycobacteria counted on the mucosal surface and recovered from macerated tissue for the three species at early time points up to 24 hours incubation. This shows that the technique of counting bacteria over the entire mucosal surface correlated well with the total number of viable bacteria on the organ culture, and that the different pathogenicity of the three species was not related to their initial interaction with airway mucosa. The significant increase in numbers of $M$ avium complex on the surface over time indicated that it survived and multiplied on the mucosal surface. Bacterial numbers calculated from macerated tissue exceeded SEM counts at 7 and 14 days, which suggests that some invasion of the tissue had also occurred. $M$ avium complex formed isolated colonylike patches and did not spread over the surface. These "colonies" were probably located at the sites of initial adherence. This suggests that there were few exposed binding sites on the mucosa for mycobacterial adherence. $M$ tuberculosis was recovered from macerated tissue in significantly increased numbers over time, but was seen on the surface of only one of six experiments after 14 days, which shows that it did not survive on the mucosal surface but had invaded the mucosa and multiplied within the tissue. $M$ smegmatis did not survive in most organ cultures; it was only seen and recovered from one organ culture after 14 days in small numbers. This organ culture, and the organ culture on which $M$ tuberculosis was observed by SEM at 14 days, contained large amounts of damaged mucosa so that large amounts of ECM were exposed, which may have provided a site for more mycobacteria to adhere than was usual.

A large increase in $M$ avium complex on the mucosal surface occurred between 7 and 14 days, suggesting that a critical number of these bacteria are required before rapid multiplication occurs, similar to the principle of "quorum sensing" reported for other bacteria. ${ }^{2930}$ Further work is required to investigate the relationship between the initial numbers of $M$ avium complex adhering to the mucosa and infection of the mucosa since the infecting dose used in this study was high. This relationship may be important in understanding which patients are vulnerable to infection, since the greater the airway damage, the greater the number of exposed adherence targets and hence the greater the risk of infection with $M$ avium complex. Such a study may also offer answers as to why $M$ smegmatis was unable to survive in the organ culture after initially adhering in similar numbers to $M$ avium complex and $M$ tuberculosis. This might relate to nutritional factors, host defences of the tissue or, possibly, the strength of adherence to the tissue.

In our previous studies we have reported that mycobacteria adhere to mucus with a fibrous appearance under SEM. ${ }^{21} 22$ The observation of $M$ avium complex and $M$ tuberculosis within submucosal glands in the present study was not made in sections where glands were seen beneath intact mucosa, suggesting that bacteria could only penetrate those glands associated with mucosal damage. This site may represent a potential route of invasion for mycobacteria. Mycobacteria were not seen within the tissue of the organ culture at other sites by light microscopy, suggesting that invasion is an infrequent event. This is not to say that a rare process is not of potential importance in the pathogenesis of mycobacterial infections, but rather indicates a possible limitation in sensitivity of the techniques employed in this study. The organ culture model well represents the initial interaction of mycobacteria with components of the mucosal surface but, over longer periods of incubation, the absence of a circulation means that the role of the immune system is limited. For example, granuloma were never observed.

Despite these limitations and those of any in vitro model, this study has made several original observations about the interaction of mycobacteria with the human respiratory mucosa. The initial adherence of each of the three species to the mucosa is similar. Mycobacteria adhere to ECM exposed in sites of mucosal damage, to mucus with a fibrous appearance, and occasionally at other sites including healthy unciliated cells. After 14 days the three species showed differences in their interaction with the tissue. $M$ avium complex multiplied on the mucosal surface in discrete colonies, $M$ tuberculosis was lost from the surface but multiplied within the tissue, and $M$ smegmatis was usually eradicated. An understanding of these differences may give important insights into the pathogenesis of mycobacterial disease. Since the results accord with the clinical features of infection by the three species, this model will be useful in further investigating the virulence of mycobacteria and determining why species differ despite identical initial interactions with the respiratory mucosa.

\section{ACKNOWLEDGEMENT}

This research was funded by a grant from the Hawley Legacy.

\section{Authors' affiliations}

A M Middleton, R Wilson, Host Defence Unit, Royal Brompton Hospital, London, UK

A M Middleton, M V Chadwick, Department of Microbiology, Royal Brompton Hospital

A G Nicholson, Department of Histopathology, Royal Brompton Hospital A Dewar, Electron Microscopy Department, Imperial College School of

Science, Technology and Medicine, London, UK

C Feldman, Department of Medicine, University of Witwatersrand, Johannesburg, South Africa

\section{REFERENCES}

1 Contreras MA, Cheung, OT, Sanders DE, et al. Pulmonary infection with nontuberculous mycobacteria. Am Rev Respir Dis 1988;137:149-52.

2 Hielte L, Petrini B, Källenius G, et al. Prospective study of mycobacterial infections in patients with cystic fibrosis. Thorax 1990;45:397-400.

3 Wilson R, Abdallah S. Pulmonary disease caused by nontuberculous mycobacteria in immunocompetent patients. In: Wilson R, ed. Tuberculosis. The European Respiratory Monograph. Volume 2. Sheffield: European Respiratory Society, 247-72.

4 Reddy VM. Mechanisms of Mycobacterium avium complex pathogenesis. Front Biosci 1998;3:D525-31.

5 Iseman MD. Mycobacterium avium complex and the normal host: the other side of the coin. N Engl J Med 1989;321:896-8

6 Prince DS, Peterson DD, Steiner RM, et al. Infection with Mycobacterium avium complex in patients without predisposing conditions. N Engl J Med 1989;321:863-8.

7 Reich JM, Johnson RE. Mycobacterium avium complex pulmonary disease presenting as an isolated lingular or middle lobe pattern: the Lady Windermere syndrome. Chest 1992;101:1605-9.

8 Epstein MD, Aranda CP, Bonk S, et al. The significance of Mycobacterium avium complex cultivation in the sputum of patients with pulmonary tuberculosis. Chest 1997;111:142-7.

9 Dhillon SS, Watanakunakorn C. Lady Windermere syndrome: middle lobe bronchiectasis and Mycobacterium avium complex infection due to voluntary cough suppression. Clin Infect Dis 2000;30:572-5.

10 Kalayiian RC, Toossi Z, Tomashefski JF Jr, et al. Pulmonary diseases due to infection by Mycobacterium avium complex in patients with AIDS. Clin Infect Dis 1995;20:1 186-94.

11 Yamazaki, Y, Kubo K, Sekiguchi M, et al. Analysis of BAL fluid in M avium-intracellulare infection in individuals without predisposing lung disease. Eur Respir J 1998;11:1227-31.

12 Powell DA, Walker DH. Nontuberculous mycobacterial endobronchitis in children. J Pediatr 1980;96:268-70.

13 Gupta SK, Katz BZ. Intrathoracic disease associated with Mycobacterium avium-intracellulare complex in otherwise healthy children: diagnostic and therapeutic considerations. Pediatrics 1994;94:741-2

14 Tierstein AS, Damsker B, Kirschner PA. Pulmonary infection with Mycobacterium avium-intracellulare: diagnosis, clinical patterns, treatment. Mt Sinai J Med 1990;57:209-15.

15 Obayashi Y, Fujita J, Suemitsu I, et al. Successive follow-up of chest computed tomography in patients with Mycobacterium avium-intracellulare complex. Respir Med 1999;93:1 1-5.

16 Stokes RW, Thorson LM, Speert DP. Nonopsonic and opsonic association of Mycobacterium tuberculosis with resident alveolar macrophages is inefficient. J Immunol 1998;160:5514-21. 
17 Thompson JR, Kent G. Occult tuberculosis endobronchitis in surgically resected lung specimens. Am Rev Tuberc Pulmon Dis 1958;77:931-9.

18 Bermudez LE, Shelton K, Young LS. Comparison of the ability of Mycobacterium avium, $M$ smegmatis and $M$ tuberculosis to invade and replicate within HEp-2 epithelial cells. Tuberc Lung Dis 1995;76:240-7.

19 Mehta PK, King CH, White EH, et al. Comparison of in vitro models for the study of Mycobacterium tuberculosis invasion and intracellular replication. Infect Immun 1996;64:2673-9.

20 Teitelbaum R, Schubert W, Gunther L, et al. The M cell as a portal of entry to the lung for bacterial pathogen Mycobacterium tuberculosis. Immunity 1999; 10:641-50.

21 Middleton AM, Chadwick MV, Nicholson AG, et al. The role of Mycobacterium avium complex fibronectin attachment protein in adherence to the human respiratory mucosa. Mol Microbiol 2000:38:381-91.

22 Middleton AM, Chadwick MV, Nicholson AG, et al. The interaction of Mycobacterium tuberculosis with human respiratory tissue in an organ culture with an air-interface. Am J Respir Crit Care Med 2001;163:A663

23 Snapper SB, Melton RE, Mustafa S, et al. Isolation and characterization of efficient plasmid transformation mutants of Mycobacterium smegmatis. Mol Microbiol 1990;4:1911-9.
24 Jackson AD, Rayner CF, Dewar A, et al. A human respiratory-tissue organ culture incorporating an air interface. Am J Respir Crit Care Med 1996; 153:1130-5.

25 Dowling RB, Rayner CFJ, Rutman A, et al. Effect of salmeterol on Pseudomonas aeruginosa infection of respiratory mucosa. Am J Respir Crit Care Med 1997; 155:327-36.

26 Soane MC, Jackson A, Maskell D, Allen A, et al. Interaction of Bordetella pertussis with human respiratory mucosa in vitro. Respir Med 2000;94:791-9.

27 Clark MA, Hirst BH, Jepson MA. M-cell surface $\beta 1$ integrin expression and invasion-mediated targeting of Yersinia pseudotuberculosis to mouse Peyer's patch $M$ cells. Infect Immun 1998;66:1237-43.

28 Snewin VA, Gares M-P, Gaora PÓ, et al. Assessment of immunity to mycobacterial infection with luciferase reporter constructs. Infect Immun 1999:67:4586-93.

29 Salmond GPC, Bycroft BW, Stewart GS, et al. The bacterial 'enigma': cracking the code of cell-cell communication. Mol Microbiol $1995 ; 16: 615-24$.

30 Dunny GM, Leonard BA. Cell-cell communication in gram positive bacteria. Am Rev Microbiol 1997;51:527-64.

\section{LUNG ALERT}

\section{Higher influenza-related paediatric admission rates in the tropics than in temperate regions \\ $\Delta$ Chiu S S, Lung Lau Y, Chan K H, et al. Influenza-related hospitalisations among children in Hong Kong. N Engl J} Med 2002;347:2097-103

I his retrospective population based study calculated the rate of excess hospital admissions due to influenza in children aged 15 or under with acute respiratory disease. The data were collected from the Hospital Authority of the Hong Kong Special Administrative Region, China. The excess admissions were calculated using an excess hospital admission model from periods of influenza peaks and baseline respiratory syncytial virus rates ( 7 weeks each in 1998 and 1999). The adjusted rates of influenza-related excess hospital admissions for 1998 and 1999 per 10000 children were 278.5 and 288.2 for children aged <1 year, 218.4 and 209.3 for those aged $1-<2$ years, 125.6 and 77.3 for those aged $2-<5$ years, 57.3 and 20.9 for children aged $5-<10$ years, and 16.4 and 8.1 for children aged $10-15$ years. Influenza-related excess admissions accounted for 38606 extra bed-days (5.5-8.2\% of all paediatric bed-days). These influenza-related hospital admission rates for children are much higher than previously published figures. Possible explanations suggested are the inclusion of high risk patients, differences in admission practice, and higher influenza attack rates in crowded communities.

The reasons for higher influenza-related hospital admission rates in this subtropical climate compared with temperate regions need further investigation. The study highlights the rationale for continued worldwide influenza surveillance, the major impact of this common infection, and the need for effective prevention.

D D Creer 